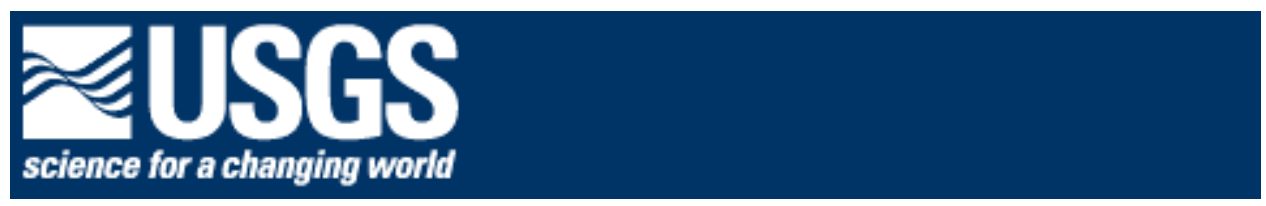

GENERATION AND MIGRATION OF PETROLEUM IN IRAQ: A 21/2D AND 3D MODELING STUDY OF JURASSIC SOURCE ROCKS

\author{
Compiled PowerPoint* Slides
}

Janet K. Pitman, Douglas, W. Steinshouer, and Michael D. Lewan

U.S. Geological Survey

Denver, CO 80225

U.S. Geological Survey Open-File Report 03-192

* Use of brand names does not constitute endorsement by the U.S. Geological Survey 


\title{
GENERATION AND MIGRATION OF PETROLEUM IN IRAQ: A 21/2D AND 3D MODELING STUDY OF JURASSIC SOURCE ROCKS
}

by Pitman, Janet $\mathrm{K}^{1}$, Steinshouer, Douglas $\mathrm{W}^{2}$, and Lewan, Michael D. ${ }^{1}$

Open-File Report

2003

This report is preliminary and has not been reviewed for conformity with the U.S. Geological Survey editorial standards or with the North American Stratigraphic Code. Any use of trade names is for descriptive purposes only and does not imply endorsement by the U.S. Government.

\author{
U.S. DEPARTMENT OF THE INTERIOR \\ U.S. GEOLOGICAL SURVEY \\ ${ }^{1}$ U.S. Geological Survey, Denver, CO 80225 \\ ${ }^{2}$ GeoLukas, Denver, CO 80203
}

\section{Preface}

The Powerpoint presentation in this report was given at the American Association of Petroleum Geologists International Meeting in Cairo, Egypt, November, 2002. Some diagrams that appeared in the original presentation have been updated in this report. It presents results of a basin modeling study of the Jurassic Petroleum System in Iraq. Basin modeling provides a strategy for optimizing exploration in frontier areas and evaluating new plays within wellexplored basins. A 1D, 21/2D, and 3D modeling study of the Jurassic petroleum system in Iraq was undertaken as part of the USGS World Energy Petroleum Resource Assessment Project in order to evaluate source-rock maturity, controls on reservoir filling, and the petroleum generation and migration history. Model simulations were generated using Integrated Exploration System's (IES) multiphase-flow (Petromod) software, a program designed to simulate multiphase fluidflow in one, two, and three dimensions. Multi-1D simulations were performed to assess the thermal evolution of the region and, in turn, maturation of the source rocks. Potential hyrocarbon migration pathways and timing of reservoir filling were evaluated based on 21/2 D (ray-tracing) simulations. 3D modeling was undertaken to visualize the distribution of reservoir accumulations and directions of hydrocarbon flow since fluid migration and reservoir filling are three-dimensional processes.

Jurassic marine shales and carbonates are the major sources of hydrocarbons produced in the Zagros basin and fold belt. A few studies have been published on the stratigraphy and sedimentology of these source rocks, but information on their burial and temperature histories and regional thermal maturation patterns are generally lacking. The timing and extent of oil generation and secondary migration also have not been adequately addressed. The present study (1) examines source rock maturities using Type II-S kerogen kinetics to describe the kerogen-tooil transformation process, (2) evaluates the timing of reservoir filling in oil fields, and (3) simulates hydrocarbon migration pathways based on the structural evolution of the basin. A better understanding of the timing and extent of oil generation, formation of traps and seals, and potential migration pathways permits simple risk assessments of areas that have potential (undiscovered) hydrocarbon resources. 


\title{
Generation and Migration of Petroleum in Iraq: A 21/2D and 3D Modeling Study of Jurassic Source Rocks
}

\author{
Janet K. Pitman \\ Douglas W. Steinshouer \\ Michael D. Lewan
}




\section{PURPOSE OF STUDY}

Determine Jurassic Source Rock Maturities and Kerogen-to-Oil Transformation Process

\# Predict Hydrocarbon Migration Pathways for Source and Reservoir Units Through Time

Evaluate Timing of Reservoir Filling in Known Fields

\# Develop Simple Risk Assessment of Undrilled Areas with Potential Hydrocarbon Resources for U.S.Geological Survey's World Petroleum Assessment 2000 (http://pubs.usgs.gov/bul/b2202-e/; U.S. Geological Survey Bulletin 2202-E) 


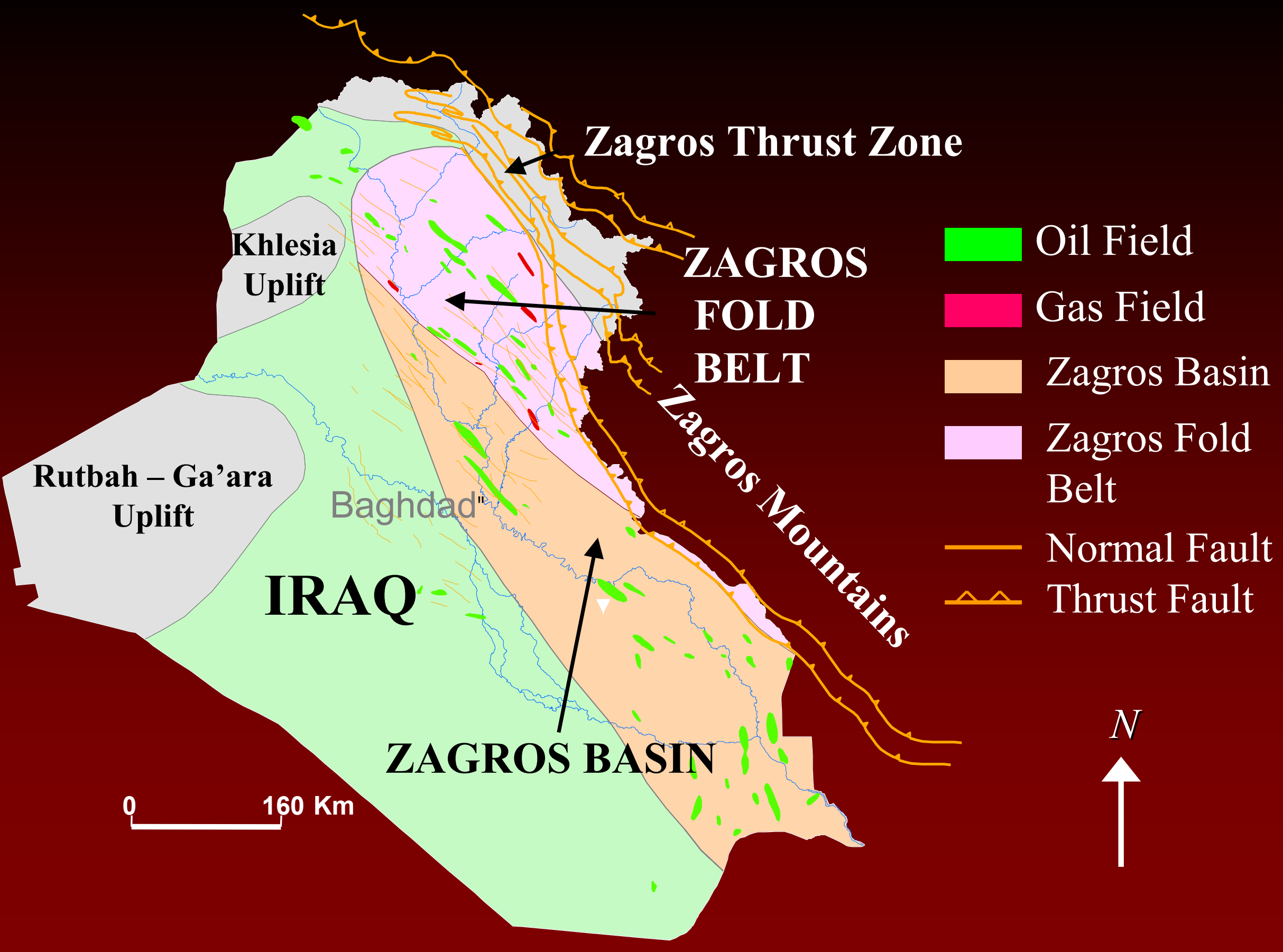




\begin{tabular}{|c|c|c|c|c|c|c|}
\hline \multicolumn{7}{|c|}{ Southern Iraq } \\
\hline $\begin{array}{l}\text { 음 } \\
\text { 똠 } \\
\text { 뭄 }\end{array}$ & $\begin{array}{l}\text { 픙 } \\
\text { 음 }\end{array}$ & 岁 & 礼 & 矛兑 & 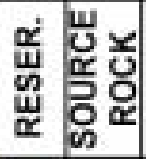 & 嵌 \\
\hline \multirow{7}{*}{ 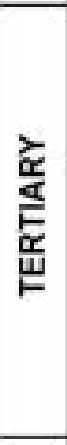 } & \multirow{3}{*}{ 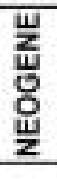 } & PLIOCENE & DIBDIBBA & & & \\
\hline & & MIOCENE & $\begin{array}{l}\text { LOWER } \\
\text { FARS }\end{array}$ & & & \\
\hline & & OLIGO. & GHAR & & & \\
\hline & \multirow{4}{*}{ 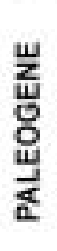 } & \multirow{3}{*}{ EOCENE } & DAMMAM & & & \\
\hline & & & RUS & \pm & & \\
\hline & & & UMM ER & & & \\
\hline & & PALEO. & RADHUMA & & & \\
\hline \multirow{16}{*}{ 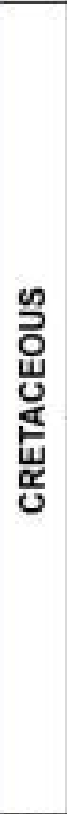 } & \multirow{9}{*}{$\begin{array}{l}\frac{x}{4} \\
\frac{0}{0} \\
\frac{0}{5}\end{array}$} & \multirow{3}{*}{$\begin{array}{c}\text { MAASTR- } \\
\text { ICHTIAN } \\
\text { TO } \\
\text { CAMPANIAN }\end{array}$} & TAYARAT & $\frac{21}{21}$ & & \\
\hline & & & SHIRANISH & $\approx=-\sim \sim \sim$ & & \\
\hline & & & HARTHA & 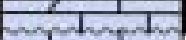 & & \\
\hline & & \multirow{2}{*}{$\begin{array}{c}\text { SANTONIAN } \\
\text { TO } \\
\text { CONLACIAN }\end{array}$} & SADI & $\frac{1}{1}$ & & \\
\hline & & & TANUSA & क्टने & & \\
\hline & & TURONIAN & MISHRIF & $1-1 \sim 1$ & & \\
\hline & & \multirow{3}{*}{ CENOMANIAN } & RUMAILA & 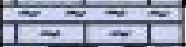 & & \\
\hline & & & AHMADI & $\sim \frac{1}{2}$ & & \\
\hline & & & MAUDDUD & $=-1--1$ & & \\
\hline & \multirow{7}{*}{$\frac{\alpha}{3}$} & ALBIAN & $\begin{array}{l}\text { NAHR } \\
\text { UMR }\end{array}$ & & & \\
\hline & & APTIAN & SHUAIBA & 111 & & \\
\hline & & BAFREMMAN & & $\because 79$ & & \\
\hline & & To & ZUBAIR & $\div \div$ & & \\
\hline & & HAUTERIVIAN & & $\div: \div$ & & \\
\hline & & VRLANGinLAN & RATAWI & $E=$ & & \\
\hline & & BEFRIASIAN & YAMAMA & 1 & & \\
\hline \multirow{4}{*}{ 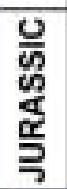 } & \multirow{4}{*}{$\begin{array}{l}\frac{\alpha}{\frac{11}{0}} \\
\frac{0}{5}\end{array}$} & TITHONIAN & SULAIY & 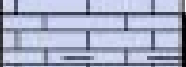 & & \\
\hline & & \multirow{3}{*}{ 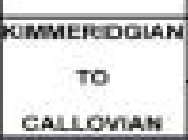 } & GOTNIA & $1 \%$ & & \\
\hline & & & 然 & $A \cdots A n$ & & \\
\hline & & & NAJMAH & 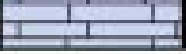 & & \\
\hline
\end{tabular}

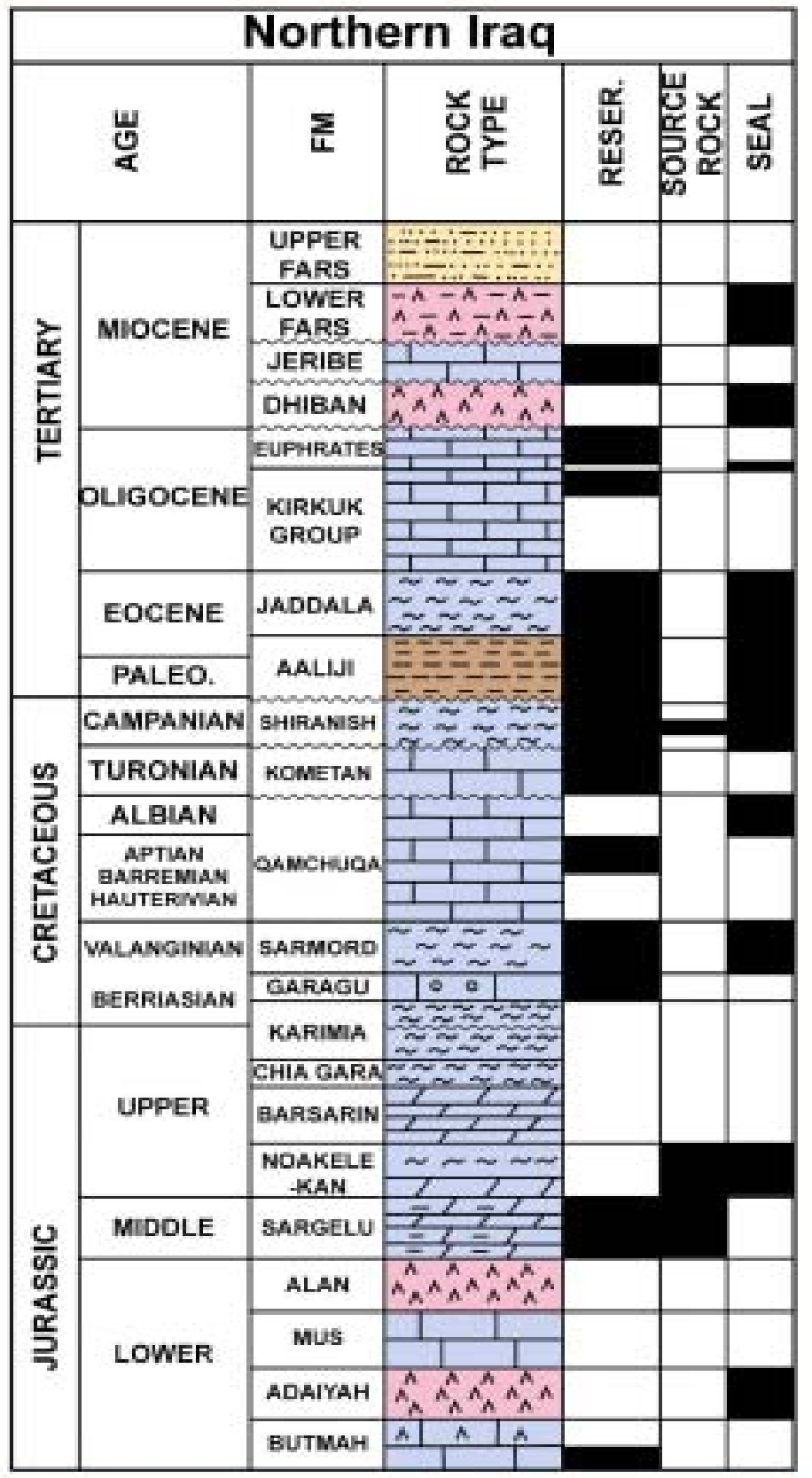

Modified from Alsharhan and Nairn (1997) 


\section{MODEL DEVELOPMENT \\ Integrated Exploration System (IES) \\ Multiphase-Flow Software}

15 Chronostratigraphic Units with Thickness, Age, Facies, and Lithology Assignments

Erosion/Nondeposition Events

\$t Temperature and Variable Heat Flow

\& Source Rock Parameters

th Fault Assignments 


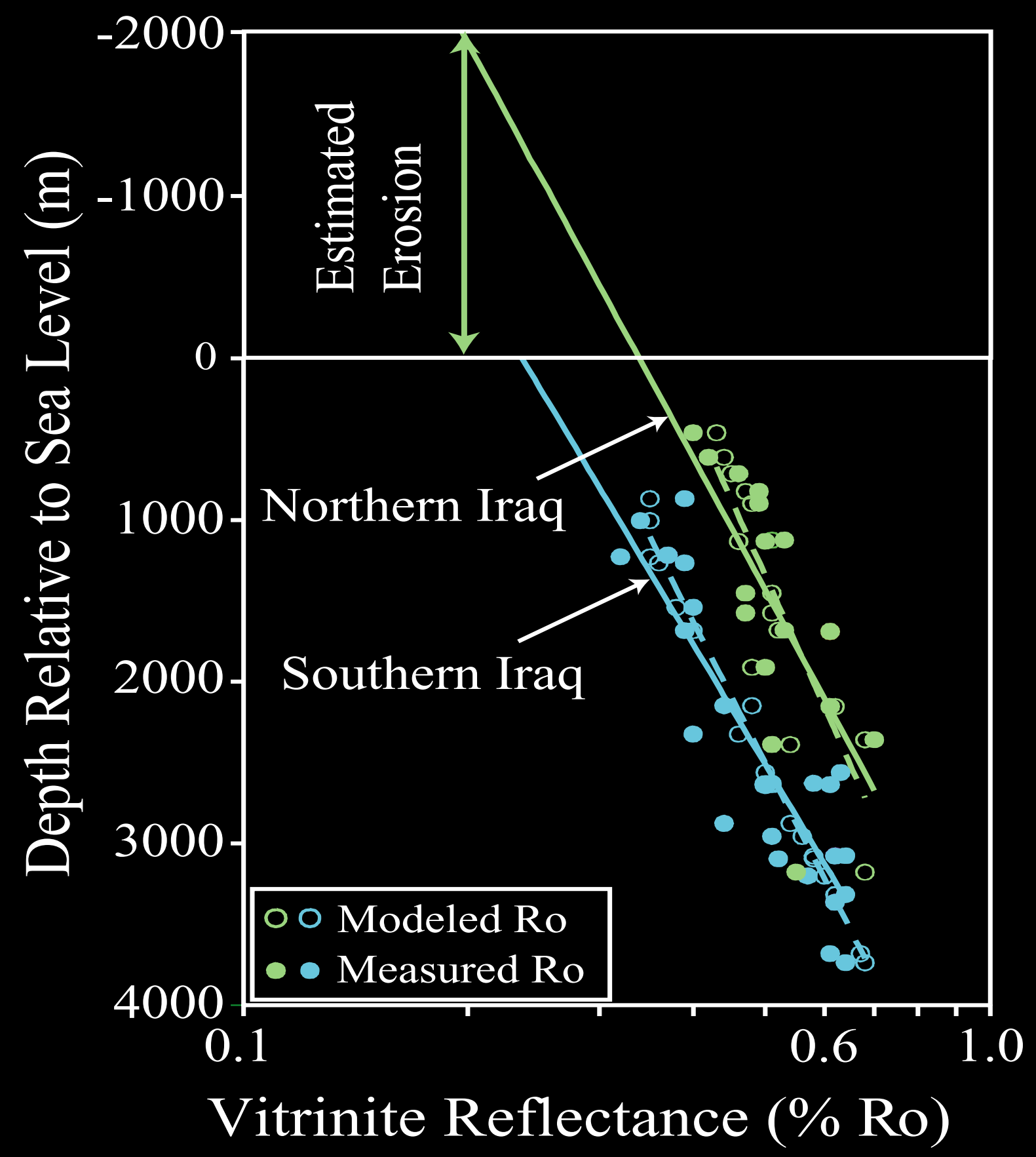




\section{SOURCE ROCK CHARACTERISTICS}

\#hickness: 10-80 m (Sargelu-Naokelekan Formations)

Organic Matter: Type II-S Kerogen

TOC $=5 \mathrm{wt}$ \% Average (Unpublished Data)

HI $=568 \mathrm{mg} / \mathrm{g}$ TOC (Unpublished Data)

N Kinetic Models: Hydrous Pyrolysis, Rock-Eval

\section{FAULT PROPERTIES}

H. Fault Age (Fold Belt): Paleogene-Neogene

Horizontal Direction: Semi-Sealing

Vertical Direction: Semi-Open

\# Major Faults: Absent in Central and Southern Zagros Basin 

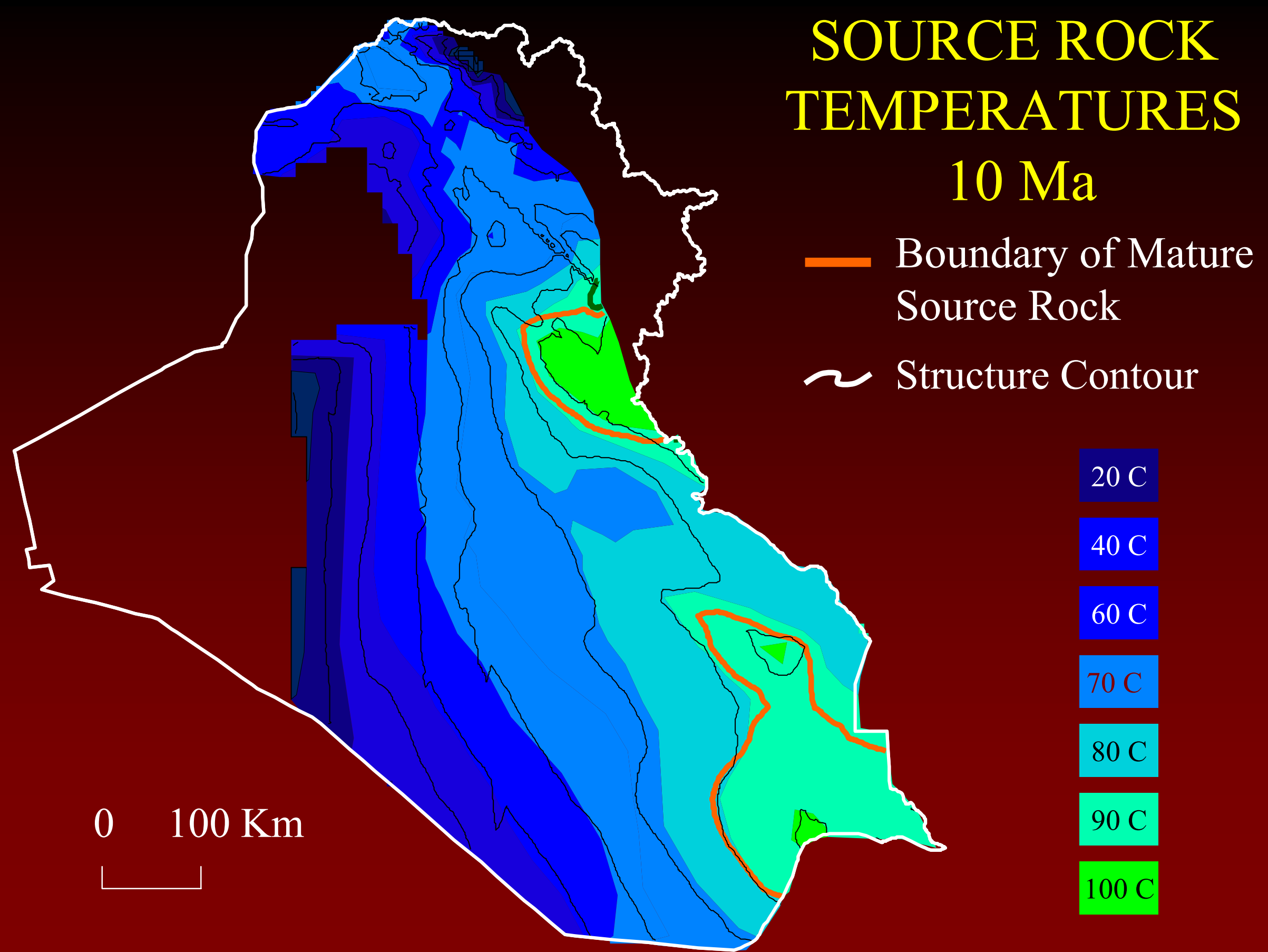

$20 \mathrm{C}$

$40 \mathrm{C}$

$60 \mathrm{C}$

$70 \mathrm{C}$

$80 \mathrm{C}$

$90 \mathrm{C}$

$100 \mathrm{C}$ 


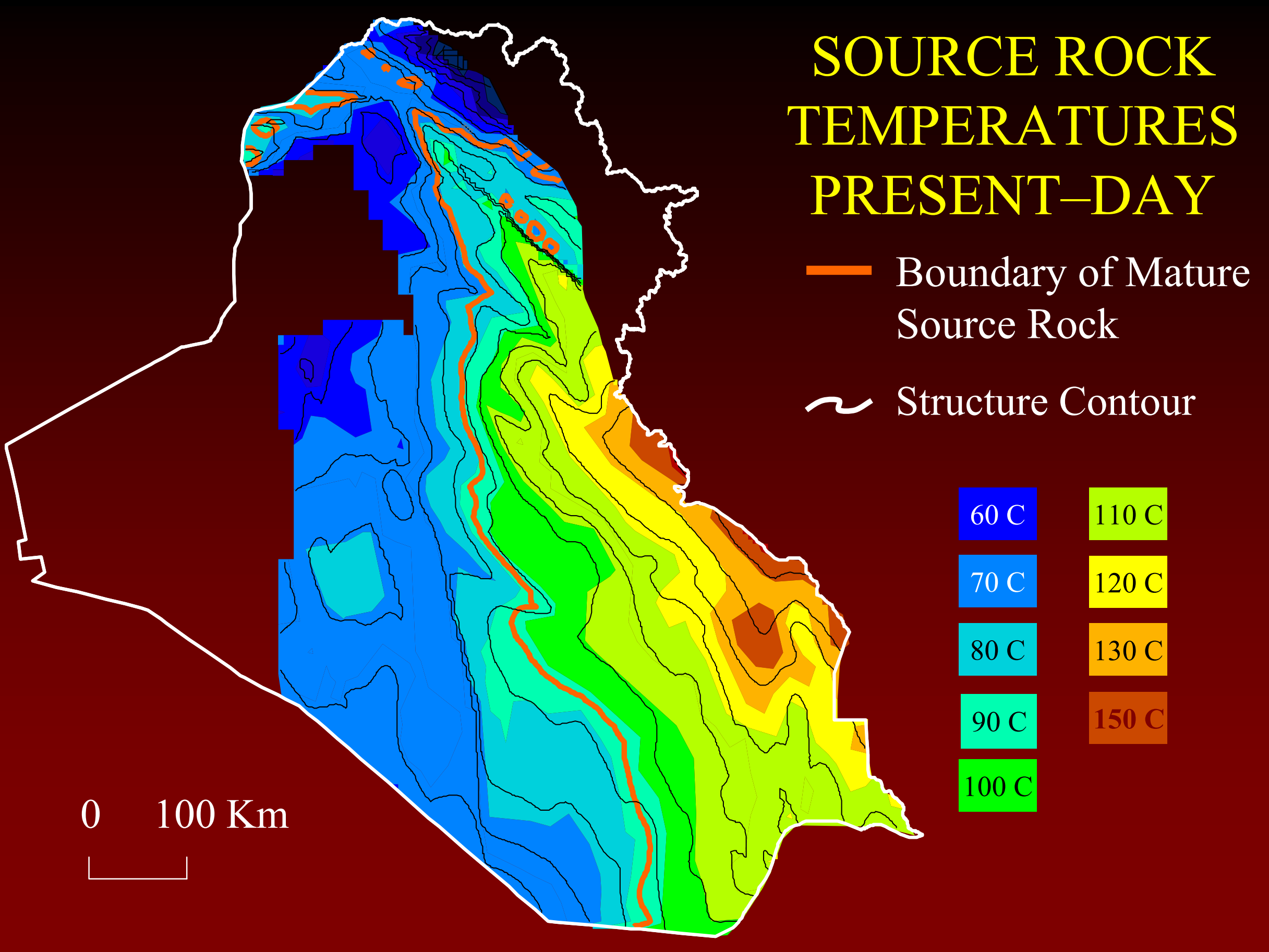




\section{ZAGROS BASIN (ZUBAIR ANTICLINE)}
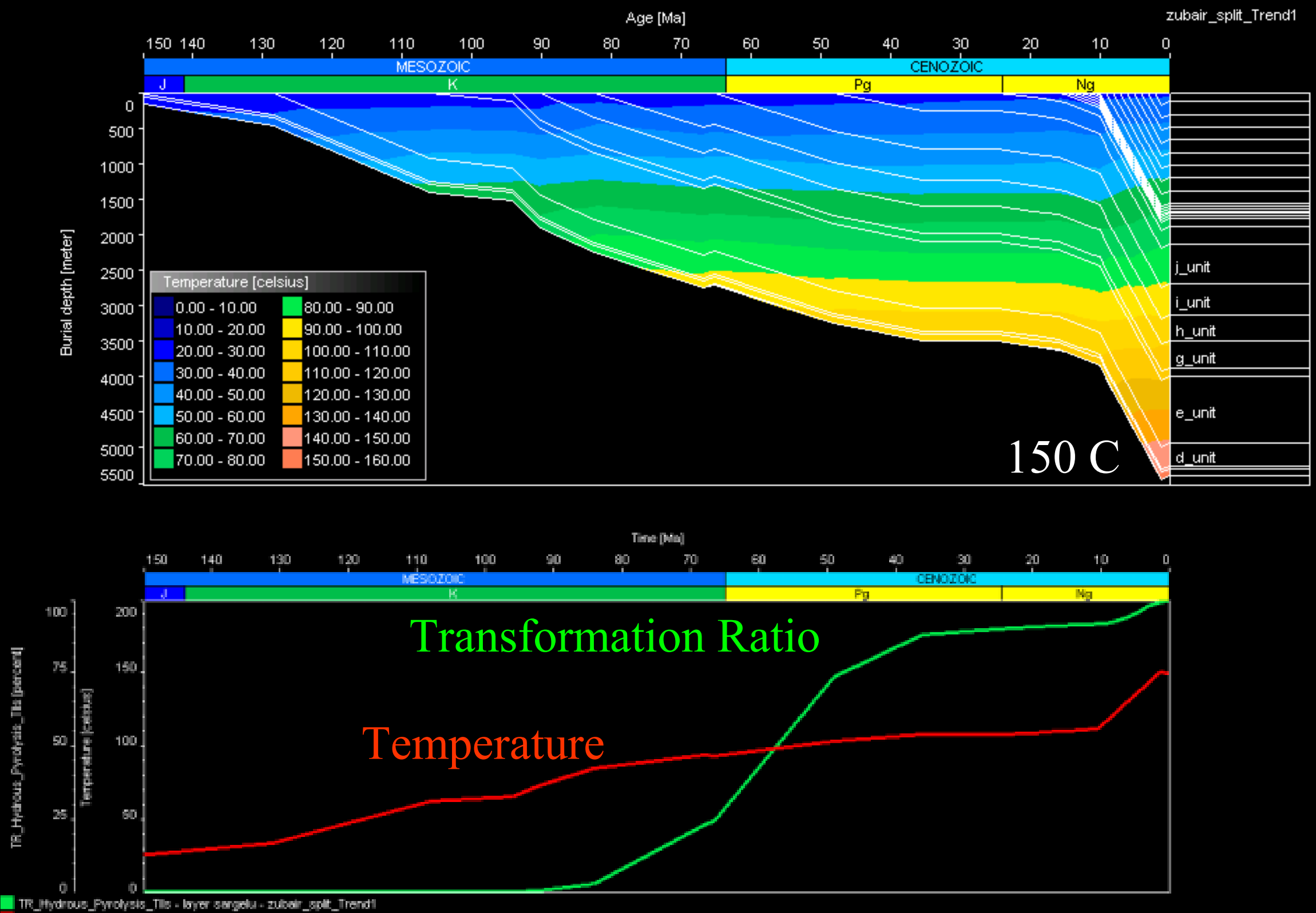


\section{FOLD BELT (KIRKUK ANTICLINE)}
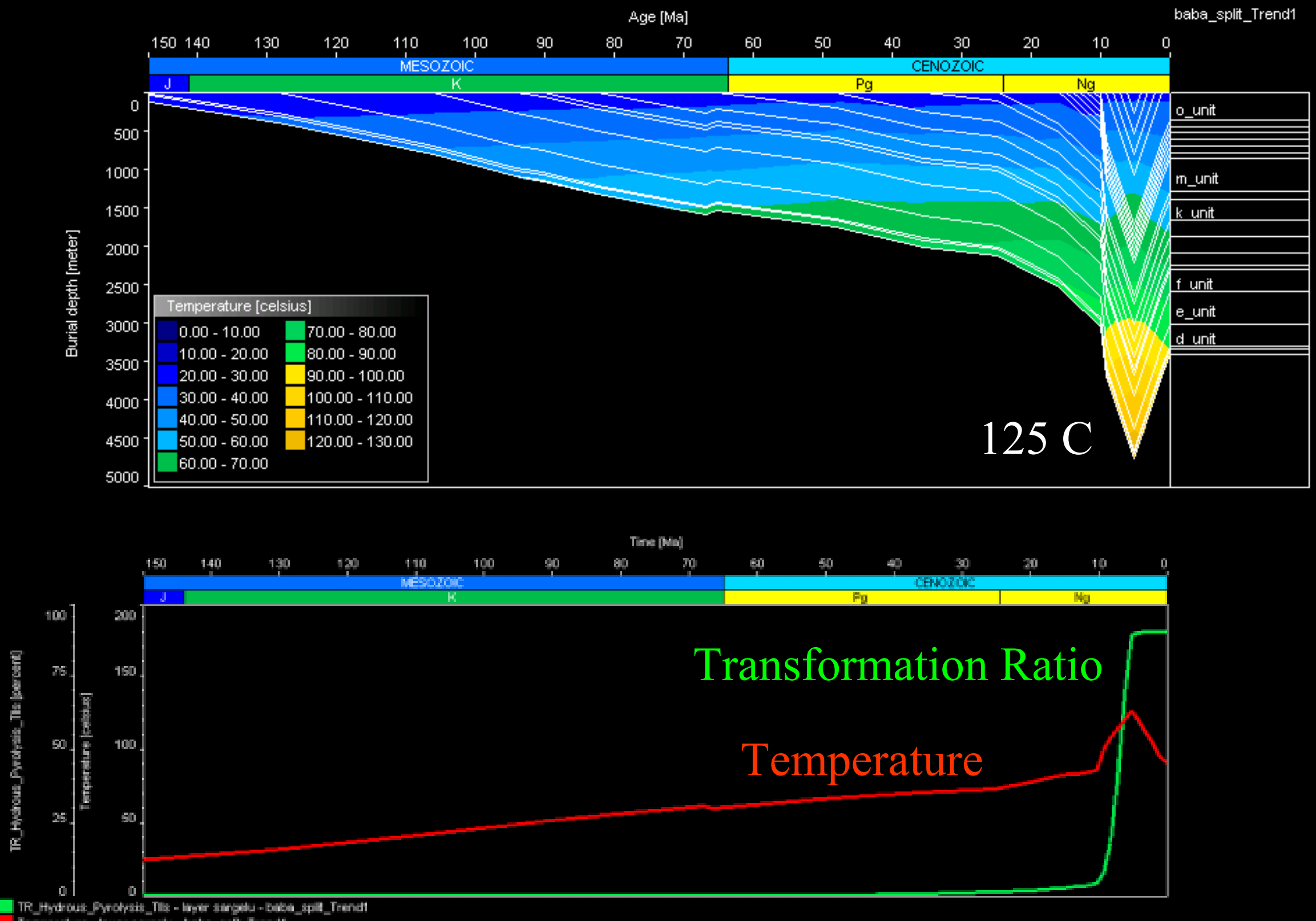


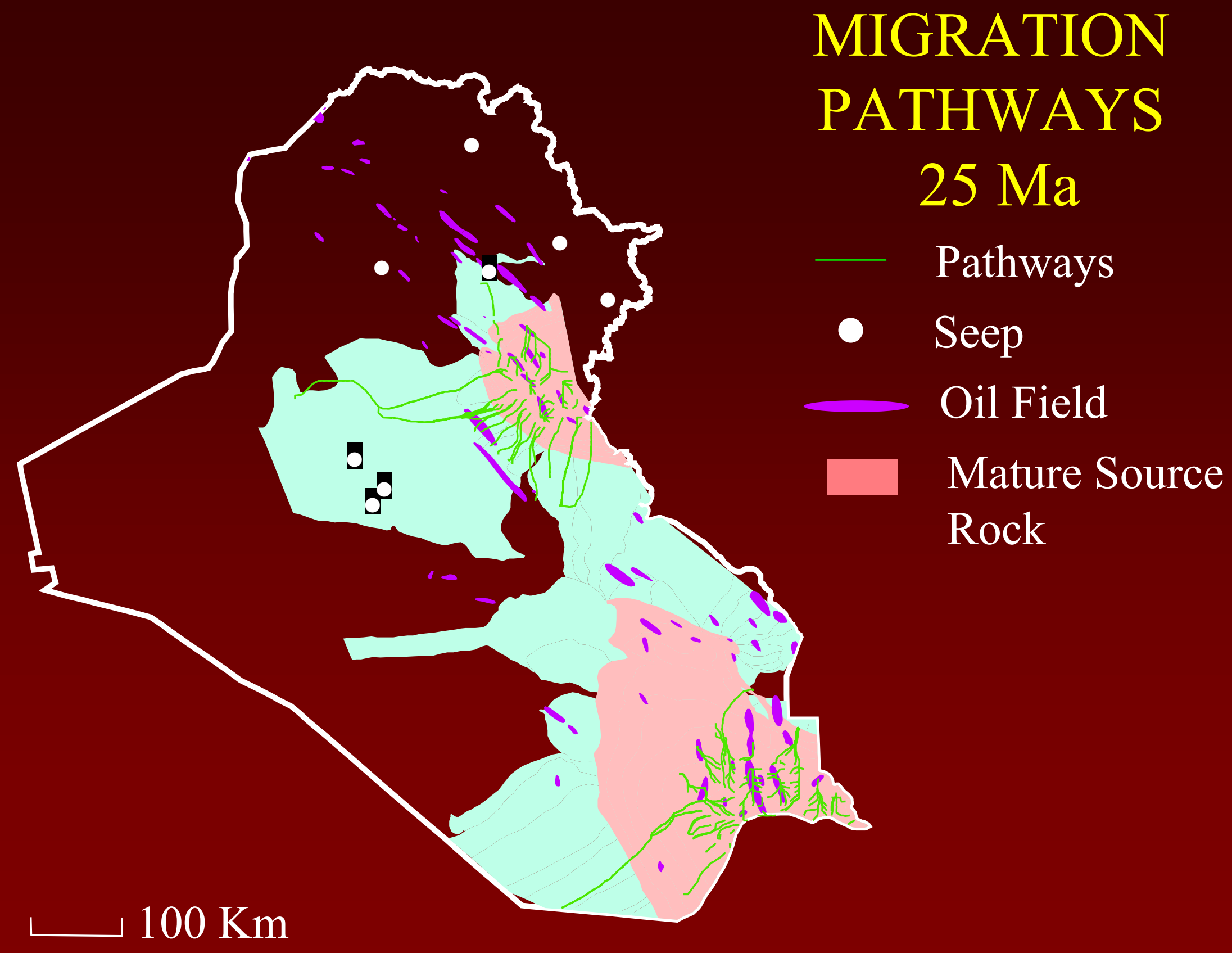




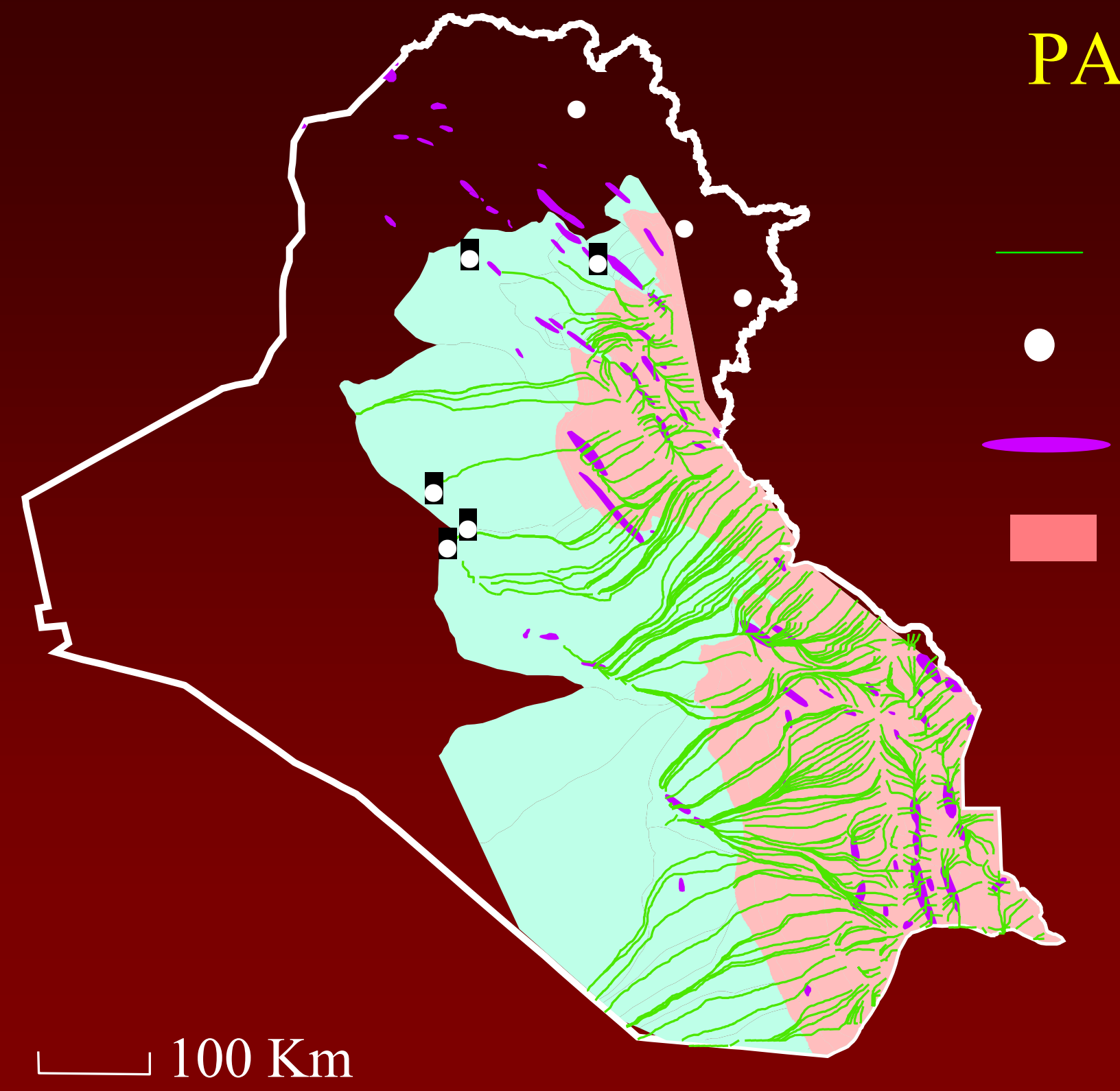

\section{MIGRATION PATHWAYS $8 \mathrm{Ma}$}

Pathways

Seep

Oil Field

Mature Source Rock 


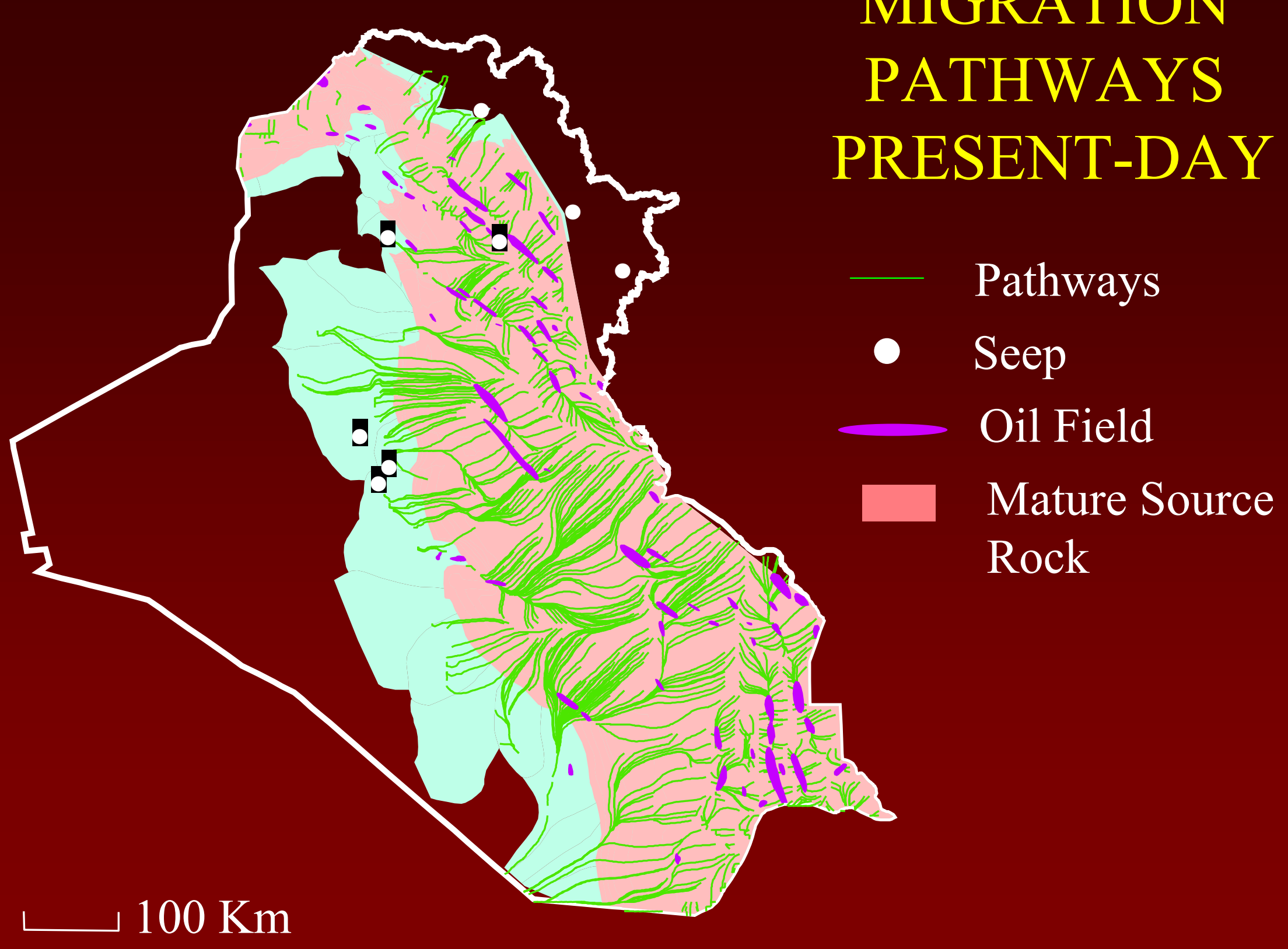


3D SIMULATION-SOUTHERN ZAGROS BASIN

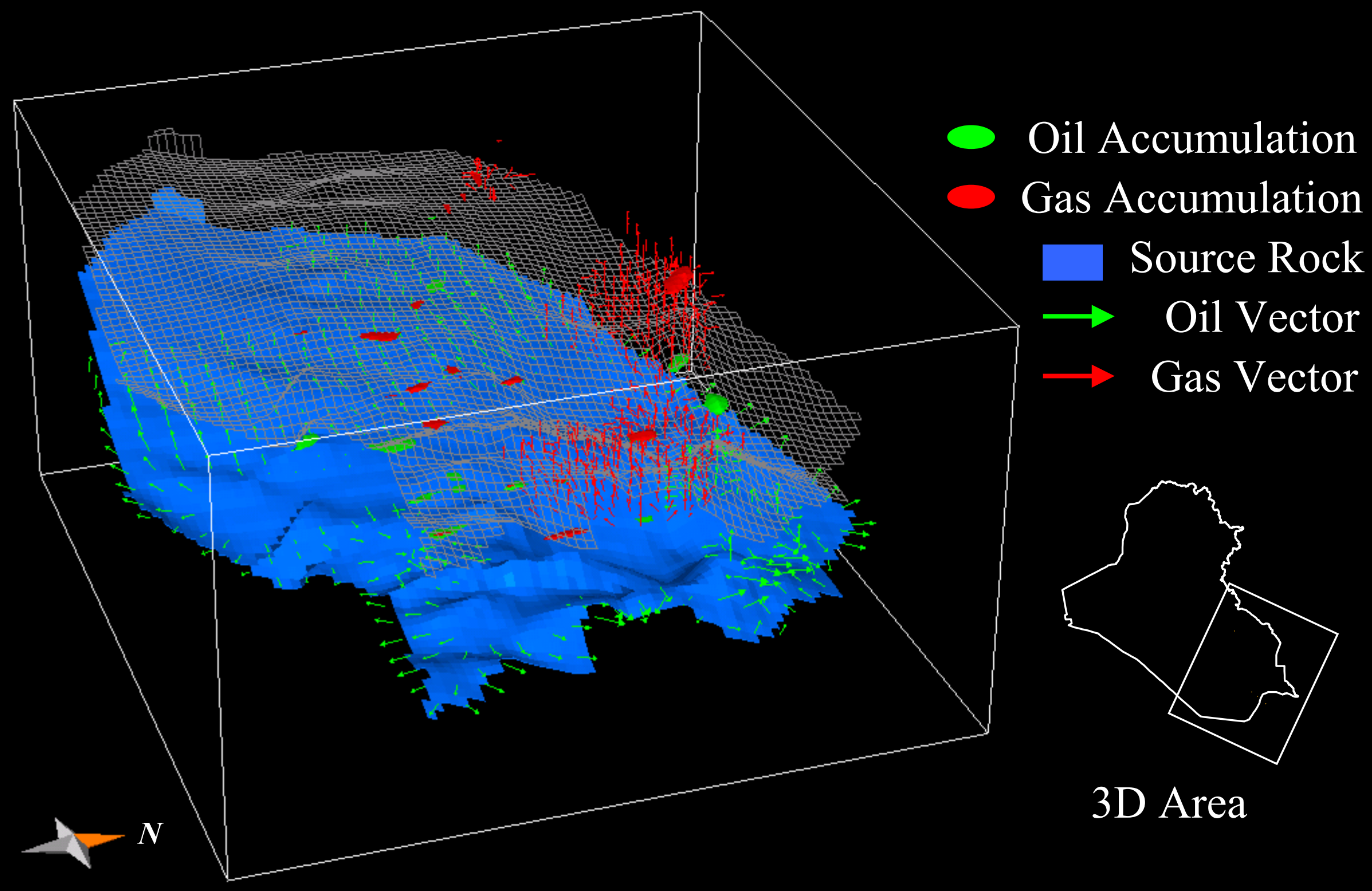




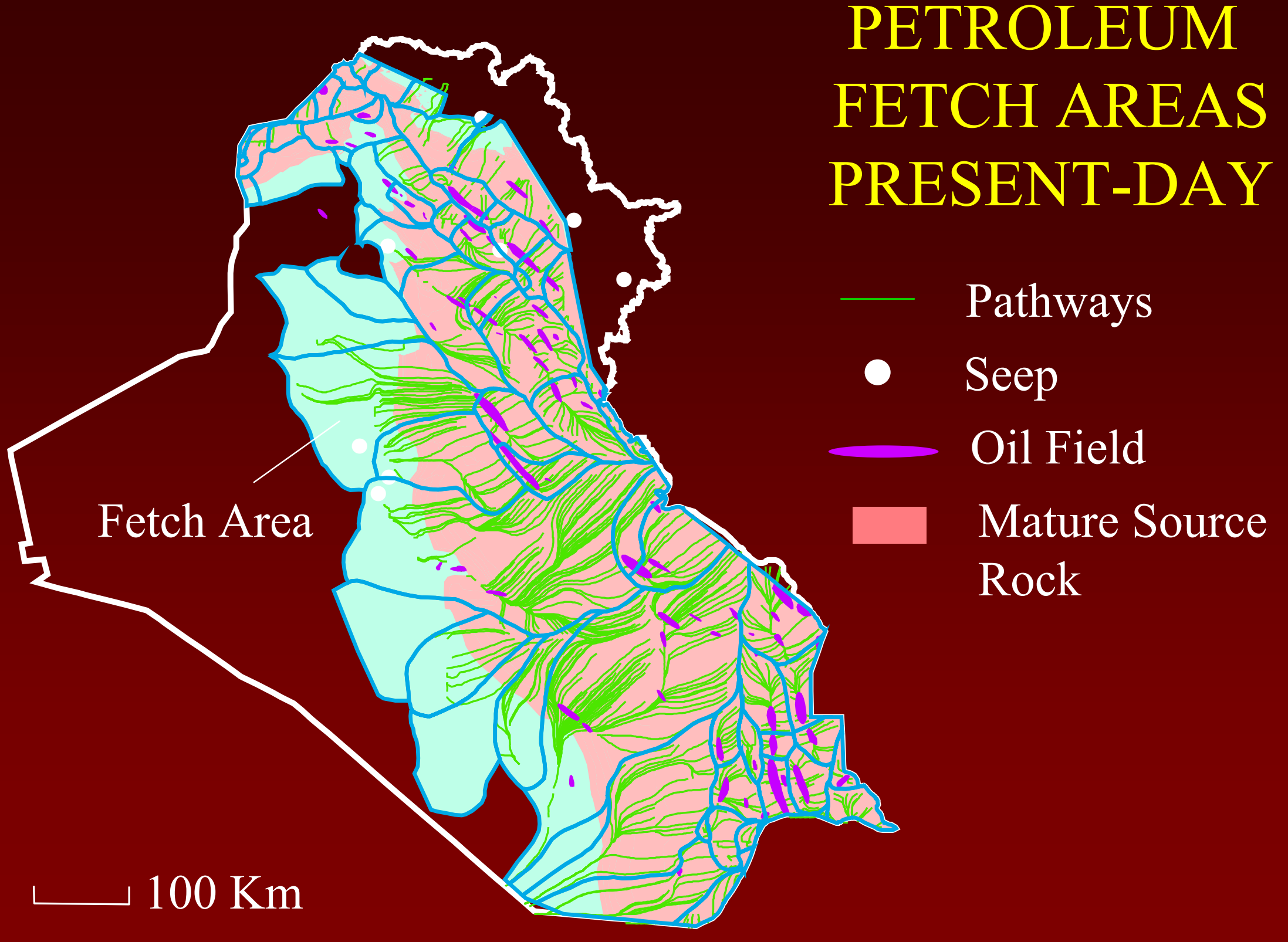




\section{CONCLUSIONS}

* Petroleum generation and migration commenced in the Late Cretaceous in the southern Zagros Basin following major trap development

\& Generation and migration in the Zagros Fold Belt began during late Paleogene/Neogene folding and faulting which resulted in large hydrocarbon loss

It Modeled migration pathways predict all known petroleum accumulations in the Basin

Anodel was used to assess prospective areas for U.S. Geological Survey's World Petroleum Assessment 2000 